\title{
ON NON-AUTONOMOUS MAXIMAL REGULARITY FOR ELLIPTIC OPERATORS IN DIVERGENCE FORM
}

\author{
PASCAL AUSCHER AND MORITZ EGERT
}

\begin{abstract}
We consider the Cauchy problem for non-autonomous forms inducing elliptic operators in divergence form with Dirichlet, Neumann, or mixed boundary conditions on an open subset $\Omega \subseteq \mathbb{R}^{n}$. We obtain maximal regularity in $L^{2}(\Omega)$ if the coefficients are bounded, uniformly elliptic, and satisfy a scale invariant bound on their fractional time-derivative of order one-half. Previous results even for such forms required control on a time-derivative of order larger than one-half.
\end{abstract}

\section{INTRODUCTION}

Let $V$ be a complex Hilbert space, $V^{*}$ be the anti-dual space of conjugate-linear functionals on $V$, and $H$ be a second complex Hilbert space in which $V$ densely embeds. Suppose that $\mathfrak{a}:[0, T] \times V \times V \rightarrow \mathbb{C}$ is a strongly measurable, bounded, quasi-coercive, non-autonomous form: Each $\mathfrak{a}(t, \cdot, \cdot)$ is a sesquilinear form on $V$ and there exist constants $\Lambda, \lambda, \eta>0$ such that

$$
|\mathfrak{a}(t, v, w)| \leq \Lambda\|v\|_{V}\|w\|_{V} \quad \text { and } \quad \operatorname{Re} \mathfrak{a}(t, v, v) \geq \lambda\|v\|_{V}^{2}-\eta\|v\|_{H}^{2}
$$

hold for all $v, w \in V$ and $t \in[0, T]$. Each form $\mathfrak{a}(t, \cdot, \cdot)$ induces a bounded operator $\mathfrak{A}(t) \in \mathcal{L}\left(V, V^{*}\right)$ via $\langle\mathfrak{A}(t) v, w\rangle=\mathfrak{a}(t, v, w)$. A classical result due to J.L. Lions states that for each $f \in \mathrm{L}^{2}\left(0, T ; V^{*}\right)$ the non-autonomous Cauchy problem

$$
u^{\prime}(t)+\mathfrak{A}(t) u(t)=f(t), \quad u(0)=0
$$

has a unique solution $u \in \mathrm{H}^{1}\left(0, T ; V^{*}\right) \cap \mathrm{L}^{2}(0, T ; V)$, see [1, p. 513]. This is usually rephrased as saying that $\mathfrak{a}$ admits maximal regularity in $V^{*}$. We remark that the original argument needs that $H$ is separable but this assumption is not necessary due to a new proof of Dier and Zacher [3, Thm. 6.1]. A famous problem, first posed explicitly by J.L. Lions in 1961 (see [6, p. 68]), concerns maximal regularity in the smaller space $H$ :

Problem 1. Is it true that for every $f \in \mathrm{L}^{2}(0, T ; H)$ the unique solution $u$ of (2) belongs to the space $\mathrm{H}^{1}(0, T ; H)$ ?

In the autonomous case $\mathfrak{A}(t)=\mathfrak{A}(0)$, de Simon proved in 1964 that maximal regularity in $H$ holds true if and only if the part of $-\mathfrak{A}(0)$ in $H$ generates a holomorphic $C_{0}$-semigroup [10]. Recent progress in the non-autonomous case has thrust

Date: December 6, 2019.

2010 Mathematics Subject Classification. Primary: 35K15. Secondary: 47A07, 26A33.

Key words and phrases. non-autonomous forms, maximal regularity, elliptic differential operators, fractional derivatives, commutator estimates.

The authors were partially supported by the ANR project "Harmonic Analysis at its Boundaries", ANR-12-BS01-0013. M.E. was supported by a public grant as part of the FMJH. 
the $\alpha$-Hölder continuity

$$
\|\mathfrak{A}(t)-\mathfrak{A}(s)\|_{V \rightarrow V^{*}} \leq C|t-s|^{\alpha} \quad(t, s \in[0, T])
$$

of $\mathfrak{A}$ into the spotlight: On the one hand Ouhabaz and Spina answered Problem 1 in the affirmative if $\mathfrak{A}$ is Hölder continuous of exponent $\alpha>\frac{1}{2}$, see [9, Thm. 3.3]. Astonishingly, Fackler on the other hand was able to construct a symmetric nonautonomous form that is $\alpha$-Hölder continuous for every $\alpha<\frac{1}{2}$ but still fails maximal regularity in $H$, see [4, Thm. 5.1]. Dier and Zacher [3] replaced the classical Hölder assumption by its square-integrated version

$$
\int_{0}^{T} \int_{0}^{T} \frac{\|\mathfrak{A}(t)-\mathfrak{A}(s)\|_{V \rightarrow V^{*}}^{2}}{|t-s|^{2 \alpha}} \frac{\mathrm{d} s \mathrm{~d} t}{|t-s|}<\infty
$$

usually referred to as fractional $\mathrm{L}^{2}$-Sobolev regularity of order $\alpha$. However, in order to prove maximal regularity in $H$ they had again to assume $\alpha>\frac{1}{2}$.

All these results left open the borderline case of $\frac{1}{2}$-regularity. In connection with his counterexample, Fackler [4] raised the question whether maximal regularity in the case $\alpha=\frac{1}{2}$ holds if $\mathfrak{a}$ induces elliptic operators in divergence form. In this note we shall provide a first positive answer to this question: If $\mathfrak{a}$ induces elliptic operators in divergence form with real symmetric coefficients, then a sufficient condition for maximal regularity in $H$ is

$$
\sup _{I \subseteq[0, T]} \frac{1}{\ell(I)} \int_{I} \int_{I} \frac{\|\mathfrak{A}(t)-\mathfrak{A}(s)\|_{V \rightarrow V^{*}}^{2}}{|t-s|} \frac{\mathrm{d} s \mathrm{~d} t}{|t-s|}<\infty
$$

where $I$ is an interval and $\ell(I)$ its length. This is the scale invariant version of Dier and Zacher's condition (4) in the borderline case $\alpha=\frac{1}{2}$, but (4) and (5) do not compare. Moreover, we obtain maximal regularity in $H$ for complex and possibly non-symmetric coefficients if we impose a condition akin to (5) directly on the coefficients. In order to state our result more precisely, we need to recall some standard notation.

1.1. Notation and precise statement of the main result. The John-Nirenberg space $\mathrm{BMO}(\mathbb{R})$ is defined as the space of locally integrable functions $f$ modulo constants that have bounded mean oscillation

$$
\|f\|_{\mathrm{BMO}(\mathbb{R})}:=\sup _{I} f_{I}\left|f(t)-f_{I}\right| \mathrm{d} t<\infty,
$$

where the supremum runs over all bounded intervals $I \subseteq \mathbb{R}$ and $f_{I}$ denotes the average on $I$. For $\alpha \in(0,1)$ the fractional $t$-derivative $D_{t}^{\alpha}$ is defined on the space $\mathcal{S}^{\prime}(\mathbb{R}) / \mathcal{P}$ of tempered distributions modulo polynomials by the Fourier symbol $|\tau|^{\alpha}$.

By a non-autonomous form $\mathfrak{a}$ inducing elliptic operators in divergence form with either Dirichlet, Neumann, or mixed boundary conditions, we always mean the following special setup: The Hilbert space $H=\mathrm{L}^{2}(\Omega)$, where $\Omega \subseteq \mathbb{R}^{n}$ is a nonempty open set, the Hilbert space $V$ is a closed subspace of the first-order Sobolev space $\mathrm{H}^{1}(\Omega)$ that contains $\mathrm{H}_{0}^{1}(\Omega)$, the closure of the test functions in $\mathrm{H}^{1}(\Omega)$, and

$$
\mathfrak{a}(t, v, w)=\int_{\Omega} A(t, x) \nabla v(x) \cdot \overline{\nabla w(x)} \mathrm{d} x,
$$

where $A:[0, T] \times \Omega \rightarrow \mathbb{C}^{n \times n}$ is a bounded and measurable function for which there exists $\Lambda, \lambda>0$ such that

$$
\lambda|\xi|^{2} \leq \operatorname{Re}(A(t, x) \xi \cdot \bar{\xi}) \quad \text { and } \quad|A(t, x) \xi \cdot \zeta| \leq \Lambda|\xi||\zeta|
$$


for all $t \in[0, T]$, a.e. $x \in \Omega$, and all $\xi, \zeta \in \mathbb{C}^{n}$. Note that such $\mathfrak{a}$ satisfies (11) with $\eta=\lambda$. The non-autonomous form introduced above induces the divergence form operators $\mathfrak{A}(t)=-\operatorname{div} A(t, \cdot) \nabla \in \mathcal{L}\left(V, V^{*}\right)$. The boundary conditions on $\partial \Omega$ are encoded in $V$ by a formal integration by parts only if one restricts to the part of $\mathfrak{A}(t)$ in $H$. In fact, this is the reason why for forms inducing differential operators the notion of maximal regularity in $H$ is so much more preferable to that of maximal regularity in $V^{*}$. Finally, we note that for any bounded form as in (6) (no need for coercivity)

$$
\|\mathfrak{A}(t)\|_{V \rightarrow V^{*}} \lesssim \operatorname{essup}_{x \in \Omega}|A(t, x)|,
$$

where here and throughout $|A(t, x)|$ denotes the norm of $A(t, x)$ as an operator on the Euclidean space $\mathbb{C}^{n}$, and that the converse estimate holds at least if $A(t, x)$ is real and symmetric for almost every $x$. The latter statement can be proved by mimicking the argument performed in 13 for the lower bound of sesquilinear forms. In particular, when applied to the differences $\mathfrak{A}(t)-\mathfrak{A}(s)$, we see that for real symmetric coefficients the abstract Hölder condition (3) is equivalent to

$$
\left.|A(t, x)-A(s, x)| \leq C|t-s|^{\alpha} \quad \text { (a.e. } x \in \Omega, s, t \in[0, T]\right) .
$$

Our main result can now be stated as follows.

Theorem 2. Consider a non-autonomous form $\mathfrak{a}$ inducing elliptic divergence-form operators with either Dirichlet, Neumann, or mixed boundary conditions on an open set $\Omega \subseteq \mathbb{R}^{n}$ as defined above. If there exists a finite $M \geq 0$ such that

$$
\sup _{I \subseteq[0, T]} \frac{1}{\ell(I)} \int_{I} \int_{I} \frac{|A(t, x)-A(s, x)|^{2}}{|t-s|^{2}} \mathrm{~d} s \mathrm{~d} t \leq M \quad(\text { a.e. } x \in \Omega),
$$

then $\mathfrak{a}$ has maximal regularity in $H=\mathrm{L}^{2}(\Omega)$. More precisely, given $f \in \mathrm{L}^{2}(0, T ; H)$, the unique solution $u \in \mathrm{H}^{1}\left(0, T ; V^{*}\right) \cap \mathrm{L}^{2}(0, T ; V)$ of problem (2) satisfies

$$
\|u\|_{\mathrm{H}^{1}(0, T ; H)}+\|u\|_{\mathrm{H}^{1 / 2}(0, T ; V)} \leq C\|f\|_{\mathrm{L}^{2}(0, T ; H)},
$$

where $C$ depends on $\lambda, \Lambda, M, T$, and $n$.

Remark 3. From the discussion above, we conclude that for real symmetric $A$ the condition (8) is even weaker than (51) as we have interchanged the essential supremum in $x$ with the integral sign. The additional regularity $u \in \mathrm{H}^{1 / 2}(0, T ; V)$ is not expected a priori, given the notion of maximal regularity in $H$. This seems to be a somewhat new phenomenon that first appeared in [3]. For background information on the vector-valued fractional Sobolev spaces the reader can refer to the appendix of [3].

We shall give the proof of Theorem 2 in Section 3 below. It relies on a reduction to the non-autonomous problem on the real line, which we shall investigate in Section 2, Therein, the $\mathrm{L}^{2}$-boundedness of commutators $\left[A(\cdot, x), D_{t}^{1 / 2}\right]$ for $x \in \Omega$ under our assumption on $A$ will be the crucial ingredient. Let us remark that commutator estimates have also been a central theme in Dier and Zacher's new approach to maximal regularity [3]. The difference is that in our special setup $A(\cdot, x)$ is valued in the finite dimensional space $\mathbb{C}^{n \times n}$. Thus, we can rely on the optimal commutator bound and do not have to waste an ' $\varepsilon$ of a derivative' as is traditional in some vector-valued extensions. 
1.2. Comparison to earlier results. Let us close the discussion by relating the regularity assumption in Theorem 2 to previously introduced conditions (in the case of real symmetric coefficients). To do so rigorously, we anticipate an extension result from Lemma 11 further below: On assuming (8), we can extend $A$ to a map $\mathbb{R} \times \Omega \rightarrow \mathbb{C}^{n \times n}$ in such a way that this estimate remains valid for every bounded interval $I \subseteq \mathbb{R}$. Being real and symmetric is a property that is preserved under this extension.

Taking this lemma for granted, the results of Strichartz [12] on BMO-Sobolev spaces yield that (8) is strictly stronger than $\frac{1}{2}$-Hölder continuity of $\mathfrak{A}$ and in fact equivalent to $A$ having an extension $A: \mathbb{R} \times \Omega \rightarrow \mathbb{C}^{n \times n}$ that satisfies

$$
\left.\left\|D_{t}^{1 / 2} A(\cdot, x)\right\|_{\mathrm{BMO}(\mathbb{R})^{n \times n}} \leq C M \quad \text { (a.e. } x \in \Omega\right) .
$$

To see how the results in [12] apply, the reader should recall that the essential supremum of $A(t)$ with respect to $x$ compares to the norm of $\mathfrak{A}(t)$. Taking into additional account the classical embeddings of Besov spaces [11, Sec. V.5.2], we obtain that (8) is strictly weaker than any of the Dini conditions

$$
\int_{0}^{T} \operatorname{essup}_{s \in \mathbb{R}}\|\mathfrak{A}(t+s)-\mathfrak{A}(s)\|_{V \rightarrow V *}^{q} \frac{\mathrm{d} t}{t^{1+q / 2}}<\infty,
$$

where $q \in[1,2]$. For $q=1$ this is the condition used by Ouhabaz and Haak [5. We also see that any of the conditions above is implied by $\alpha$-Hölder continuity for an $\alpha>\frac{1}{2}$. Coming up with a particular example, an admissible function in product form $A(t, x)=1+|t|^{1 / 2} A(x)$ will not satisfy any of the Dini conditions but it does satisfy (8) since $D_{t}^{1 / 2}|t|^{1 / 2}=\log |t|$ is a BMO-function on the real line.

\section{The NON-AUtonomous PROBlem on the REAL Line}

We begin by investigating the non-autonomous problem on the real line. So, following our previously introduced notation on forms inducing elliptic operators in divergence form, we assume that $A: \mathbb{R} \times \Omega \rightarrow \mathbb{C}^{n \times n}$ is bounded, measurable, and coercive in the sense that

$$
\lambda|\xi|^{2} \leq \operatorname{Re}(A(t, x) \xi \cdot \bar{\xi}) \quad \text { and } \quad|A(t, x) \xi \cdot \zeta| \leq \Lambda|\xi||\zeta|
$$

hold for all $t \in \mathbb{R}$, a.e. $x \in \Omega$, and all $\xi, \zeta \in \mathbb{C}^{n}$. We let $\mathfrak{a}: \mathbb{R} \times V \times V \rightarrow \mathbb{C}$ be the corresponding non-autonomous form defined as in (6). Next, $\nabla_{V}: V \rightarrow \mathrm{L}^{2}(\Omega)^{n}$ denotes the gradient operator defined on $V$ and $\nabla_{V}^{*}: \mathrm{L}^{2}(\Omega)^{n} \rightarrow V^{*}$ is its adjoint. As a matter of fact, $\mathfrak{A}(t)=\nabla_{V}^{*} A(t, \cdot) \nabla_{V}$. Here, and throughout, we identify $A(t, \cdot)$ with the corresponding multiplication operator on $\mathrm{L}^{2}(\Omega)^{n}$.

In the following we write $\mathrm{H}^{1 / 2}(\mathbb{R} ; H)$ for the Hilbert space of all $u \in \mathrm{L}^{2}(\mathbb{R} ; H)$ with $D_{t}^{1 / 2} u \in \mathrm{L}^{2}(\mathbb{R} ; H)$, keeping in mind that $H=\mathrm{L}^{2}(\Omega)$. We define the 'energy space'

$$
\mathrm{E}:=\mathrm{H}^{1 / 2}(\mathbb{R} ; H) \cap \mathrm{L}^{2}(\mathbb{R} ; V)
$$

equipped with the Hilbertian norm

$$
\|u\|_{\mathrm{E}}^{2}:=\int_{\mathbb{R}}\|u(t)\|_{\mathrm{L}^{2}(\Omega)}^{2}+\left\|D_{t}^{1 / 2} u(t)\right\|_{\mathrm{L}^{2}(\Omega)}^{2}+\left\|\nabla_{V} u(t)\right\|_{\mathrm{L}^{2}(\Omega)^{n}}^{2} \mathrm{~d} t .
$$

From Plancherel's theorem we obtain $\mathrm{H}^{1}\left(\mathbb{R} ; V^{*}\right) \cap \mathrm{L}^{2}(\mathbb{R} ; V) \subseteq \mathrm{E}$ with continuous embedding. By density of this embedding (note that $\mathrm{H}^{1}(\mathbb{R} ; V) \subseteq \mathrm{E}$ ) the bounded 
parabolic operator

$$
\mathfrak{L}: \mathrm{H}^{1}\left(\mathbb{R} ; V^{*}\right) \cap \mathrm{L}^{2}(\mathbb{R} ; V) \rightarrow \mathrm{L}^{2}\left(\mathbb{R} ; V^{*}\right), \quad \mathfrak{L} u:=u^{\prime}+\mathfrak{A} u
$$

naturally extends its action to a bounded operator $\mathrm{E} \rightarrow \mathrm{E}^{*}$, also denoted by $\mathfrak{L}$, via

$$
\mathfrak{L}(u)(w)=\int_{\mathbb{R}}-\left(D_{t}^{1 / 2} u \mid D_{t}^{1 / 2} H_{t} w\right)_{H}+\langle\mathfrak{A} u, w\rangle \mathrm{d} t \quad(u, w \in \mathrm{E}) .
$$

Here, $H_{t}$ denotes the Hilbert transform defined on $\mathrm{L}^{2}(\mathbb{R} ; H)$ by the Fourier symbol $i \operatorname{sgn}(\tau)$. Besides other things, the next lemma shows that the part of $\mathfrak{L}$ in $\mathrm{L}^{2}(\mathbb{R} ; H)$ is maximal accretive. We consider this an easy though fundamental observation in the field of non-autonomous parabolic problems. It implicitly appeared in [3, 8 , without being mentioned.

Lemma 4. Let $\theta \in \mathbb{C}$ with $\operatorname{Re} \theta>0$. The following assertions hold.

(i) For each $f \in \mathrm{E}^{*}$ there exists a unique $u \in \mathrm{E}$ such that $(\theta+\mathfrak{L}) u=f$. Moreover,

$$
\|u\|_{\mathrm{E}} \leq \sqrt{2} \max \left\{\frac{\Lambda+1}{\lambda}, \frac{|\operatorname{Im} \theta|+1}{\operatorname{Re} \theta}\right\}\|f\|_{\mathrm{E}^{*}} .
$$

(ii) If in addition $f \in \mathrm{L}^{2}\left(\mathbb{R} ; V^{*}\right)$, then $u \in \mathrm{H}^{1}\left(\mathbb{R} ; V^{*}\right)$ and $u$ is the unique solution of the non-autonomous problem

$$
u^{\prime}(t)+\theta u(t)+\mathfrak{A}(t) u(t)=f(t) \quad(t \in \mathbb{R})
$$

in the class $\mathrm{H}^{1}\left(\mathbb{R} ; V^{*}\right) \cap \mathrm{L}^{2}(\mathbb{R} ; V)$.

(iii) If even $f \in \mathrm{L}^{2}(\mathbb{R} ; H)$, then

$$
\|u\|_{\mathrm{L}^{2}(\mathbb{R} ; H)} \leq \frac{1}{\operatorname{Re} \theta}\|f\|_{\mathrm{L}^{2}(\mathbb{R} ; H)} .
$$

In particular, the part of $\mathfrak{L}$ in $\mathrm{L}^{2}(\mathbb{R} ; H)$ is maximal accretive with domain

$$
\mathrm{D}=\left\{u \in \mathrm{H}^{1}\left(\mathbb{R} ; V^{*}\right) \cap \mathrm{L}^{2}(\mathbb{R} ; V): \mathfrak{L} u \in \mathrm{L}^{2}(\mathbb{R} ; H)\right\} .
$$

Proof. The proof following [3,8, relies on some hidden coercivity of the parabolic operator $\mathfrak{L}$. It can be revealed using the Hilbert transform $H_{t}$. We define the sesquilinear form $\mathfrak{e}: \mathrm{E} \times \mathrm{E} \rightarrow \mathbb{C}$ by

$$
\mathfrak{e}(v, w)=\int_{\mathbb{R}}-\left(D_{t}^{1 / 2} v \mid D_{t}^{1 / 2} H_{t}\left(1+\delta H_{t}\right) w\right)_{H}+\left\langle(\theta+\mathfrak{A}) v,\left(1+\delta H_{t}\right) w\right\rangle \mathrm{d} t,
$$

with $\delta>0$ still to be chosen. Clearly $\mathfrak{e}$ is bounded. Since $H_{t}$ is skew-adjoint,

$$
\operatorname{Re} \int_{\mathbb{R}}\left(f \mid H_{t} f\right)_{H} \mathrm{~d} t=0 \quad\left(f \in \mathrm{L}^{2}(\mathbb{R} ; H)\right) .
$$

Using this along with the ellipticity of $\mathfrak{a}$, we find for all $v \in \mathrm{E}$ that

$\operatorname{Re} e(v, v) \geq \int_{\mathbb{R}} \delta\left\|D_{t}^{1 / 2} v\right\|_{\mathrm{L}^{2}(\Omega)}^{2}+(\lambda-\delta \Lambda)\|\nabla v\|_{\mathrm{L}^{2}(\Omega)^{n}}^{2}+(\operatorname{Re} \theta-\delta|\operatorname{Im} \theta|)\|v\|_{\mathrm{L}^{2}(\Omega)}^{2} \mathrm{~d} t$.

Choosing $\delta$ such that the factors in front of the second and third term are no less than $\delta$, we obtain the coercivity estimate

$$
\operatorname{Re} \mathfrak{e}(v, v) \geq \min \left\{\frac{\lambda}{\Lambda+1}, \frac{\operatorname{Re} \theta}{|\operatorname{Im} \theta|+1}\right\}\|v\|_{\mathrm{E}}^{2} \quad(v \in \mathrm{E}) .
$$


The Lax-Milgram lemma yields for each $f \in \mathrm{E}^{*}$ a unique $u \in \mathrm{E}$ with bound as required in (i) such that

$$
\mathfrak{e}(u, w)=f\left(\left(1+\delta H_{t}\right) w\right) \quad(w \in \mathbf{E}) .
$$

Since $\delta<1$, Plancherel's theorem yields that $1+\delta H_{t}$ is an isomorphism on E. Thus,

$$
\int_{\mathbb{R}}-\left(D_{t}^{1 / 2} u \mid D_{t}^{1 / 2} H_{t} w\right)_{H}+\langle(\theta+\mathfrak{A}) u, w\rangle \mathrm{d} t=f(w) \quad(w \in \mathrm{E}),
$$

that is, $(\theta+\mathfrak{L}) u=f$. This completes the proof of (i). If in addition $f \in \mathrm{L}^{2}\left(\mathbb{R} ; V^{*}\right)$, then the previous identity with $w \in \mathrm{H}^{1}(\mathbb{R} ; V)$ rewrites

$$
\int_{\mathbb{R}}-\left\langle u, w^{\prime}\right\rangle+\langle(\theta+\mathfrak{A}) u, w\rangle \mathrm{d} t=\int_{\mathbb{R}}\langle f, w\rangle \mathrm{d} t,
$$

thereby proving $u \in \mathrm{H}^{1}\left(\mathbb{R} ; V^{*}\right)$. As $\mathrm{H}^{1}(\mathbb{R} ; V)$ is dense in $\mathrm{L}^{2}(\mathbb{R} ; V)$, we have $u^{\prime}+$ $\theta u+\mathfrak{A} u=f$ as a pointwise equality in $\mathrm{L}^{2}\left(\mathbb{R} ; V^{*}\right)$. Since $\mathrm{H}^{1}\left(\mathbb{R} ; V^{*}\right) \cap \mathrm{L}^{2}(\mathbb{R} ; V) \subseteq \mathrm{E}$, the first part of the proof gives of course uniqueness of $u$ in the smaller space. This proves (ii).

Finally, we prove (iii). From the first two items we can infer that $\theta+\mathfrak{L}$ : $\mathrm{D} \rightarrow \mathrm{L}^{2}(\mathbb{R} ; H)$ is one-to-one. In order to check the resolvent estimate required for maximal accretivity, let $f \in \mathrm{L}^{2}(\mathbb{R} ; H)$. Then, by accretivity of $A$ and skewadjointness of the Hilbert transform

$$
\begin{aligned}
\operatorname{Re} \theta\|u\|_{\mathrm{L}^{2}(\mathbb{R} ; H)}^{2} & \leq \operatorname{Re} \int_{\mathbb{R}}-\left(D_{t}^{1 / 2} u \mid H_{t} D_{t}^{1 / 2} u\right)_{H}+\langle(\theta+\mathfrak{A}) u, u\rangle \mathrm{d} t \\
& =\operatorname{Re} \int_{\mathbb{R}}(f \mid u)_{H} \mathrm{~d} t \leq\|f\|_{\mathrm{L}^{2}(\mathbb{R} ; H)}\|u\|_{\mathrm{L}^{2}(\mathbb{R} ; H)} .
\end{aligned}
$$

Next, we provide an easy 'improved time-regularity result' under the additional assumption that $A$ satisfies the Lipschitz condition

$$
|A(t, x)-A(s, x)| \leq C|t-s| \quad \text { (a.e. } x \in \Omega, s, t \in \mathbb{R})
$$

for some $C>0$. Later we will apply this result qualitatively to smoothened versions of $A$ in order to justify some of our calculations.

Lemma 5. Let $f \in \mathrm{H}^{1}\left(\mathbb{R} ; V^{*}\right)$ and suppose that $A$ satisfies the Lipschitz condition. Then the solution $u=(1+\mathfrak{L})^{-1} f$ provided by Lemma 4 belongs to $\mathrm{H}^{1}(\mathbb{R} ; V)$.

Proof. The proof is a straightforward application of the method of difference quotients. For $h \in \mathbb{R}$ define

$$
D_{h} u(t)=\frac{1}{h}(u(t+h)-u(t)) \quad(t \in \mathbb{R})
$$

and similarly define $D_{h} f$ and $D_{h} \mathfrak{A}$. Since $u^{\prime}+u+\mathfrak{A} u=f$ on the real line, subtracting the equations for $u(t+h)$ and $u(t)$ shows that $D_{h} u$ is the unique solution of

$$
\left(D_{h} u\right)^{\prime}(t)+D_{h} u(t)+\mathfrak{A}(t) D_{h} u(t)=D_{h} f(t)-D_{h} \mathfrak{A}(t) u(t+h)
$$

in $\mathrm{H}^{1}\left(\mathbb{R} ; V^{*}\right) \cap \mathrm{L}^{2}(\mathbb{R} ; V)$. The right-hand side can be bounded in $\mathrm{L}^{2}\left(\mathbb{R} ; V^{*}\right)$ uniformly in $h$ as we obtain

$$
\int_{\mathbb{R}}\left\|D_{h} f(t)\right\|_{V^{*}}^{2} \mathrm{~d} t \leq \int_{\mathbb{R}}\left\|f^{\prime}(t)\right\|_{V^{*}}^{2} \mathrm{~d} t
$$


and

$$
\int_{\mathbb{R}}\left\|D_{h} \mathfrak{A}(t) u(t+h)\right\|_{V^{*}}^{2} \mathrm{~d} t \leq C^{2} \int_{\mathbb{R}}\|u(t)\|_{V}^{2} \mathrm{~d} t
$$

from our assumptions. Since $1+\mathfrak{L}: E \rightarrow E^{*}$ is an isomorphism, see Lemma 4, the norm $\left\|D_{h} u\right\|_{L^{2}(\mathbb{R} ; V)}$ can be bounded uniformly in $h$ as well. Hence, $\left\{D_{h} u\right\}_{h \in \mathbb{R}}$ has a weak limit point $v \in \mathrm{L}^{2}(\mathbb{R} ; V)$ which straightforwardly reveals itself as the weak derivative of $u$.

We continue by quoting a commutator estimate due to Murray [7, Thm. 3.3]. The reader may also see it as a consequence of the famous $T(1)$-theorem of David and Journé [2].

Proposition 6. Let $a: \mathbb{R} \rightarrow \mathbb{R}$ be bounded and Lipschitz continuous. Then the commutator $\left[a, D_{t}^{1 / 2}\right]=a D_{t}^{1 / 2}-D_{t}^{1 / 2}$ a extends from $\mathrm{H}^{1 / 2}(\mathbb{R})$ to a bounded operator on $\mathrm{L}^{2}(\mathbb{R})$ if and only if $D_{t}^{1 / 2} a \in \mathrm{BMO}(\mathbb{R})$. In this case its norm is controlled by $\left\|D_{t}^{1 / 2} a\right\|_{\mathrm{BMO}(\mathbb{R})}$.

Since $\mathrm{L}^{2}(\mathbb{R} ; H)=\mathrm{L}^{2}\left(\Omega ; \mathrm{L}^{2}(\mathbb{R})\right)$ by Fubini's theorem, we obtain a similar result on $\mathrm{L}^{2}(\mathbb{R} ; H)$ for free. This is the part of the argument that crucially uses that $H$ is not an arbitrary Hilbert space.

Corollary 7. Suppose that A satisfies the Lipschitz condition and that there exists a constant $M^{\natural}$ such that $\left\|D_{t}^{1 / 2} A(\cdot, x)\right\|_{\mathrm{BMO}(\mathbb{R})^{n \times n}} \leq M^{\natural}$ for a.e. $x \in \Omega$. Then the commutator estimate

$$
\left\|\left[A, D_{t}^{1 / 2}\right] u\right\|_{\mathrm{L}^{2}(\mathbb{R} ; H)^{n}} \leq C M^{\natural}\|u\|_{\mathrm{L}^{2}(\mathbb{R} ; H)^{n}} \quad\left(u \in \mathrm{H}^{1 / 2}(\mathbb{R} ; H)^{n}\right)
$$

holds true with $C$ depending only on the dimension $n$.

Proof. If $u \in \mathrm{H}^{1 / 2}(\mathbb{R} ; H)^{n}$, then $u(\cdot, x) \in \mathrm{H}^{1 / 2}(\mathbb{R})^{n}$ for a.e. $x \in \Omega$. Hence, the commutator $\left[A(\cdot, x), D_{t}^{1 / 2}\right] u(\cdot, x)$ is a priori defined and the claim follows on applying Murray's estimate coordinate-wise and integrating with respect to $x$.

We are in a position to prove our main result on maximal regularity of the non-autonomous problem on the real line.

Proposition 8. If there is a constant $M^{\natural}$ such that $\left\|D_{t}^{1 / 2} A(\cdot, x)\right\|_{\operatorname{BMO}(\mathbb{R})^{n \times n}} \leq M^{\natural}$ for a.e. $x \in \Omega$, then for every $f \in \mathrm{L}^{2}(\mathbb{R} ; H)$ the solution $u=(1+\mathfrak{L})^{-1} f$ of the non-autonomous problem

$$
u^{\prime}(t)+u(t)+\mathfrak{A}(t) u(t)=f(t) \quad(t \in \mathbb{R})
$$

belongs to $\mathrm{H}^{1}(\mathbb{R} ; H)$. More precisely, for some $C>0$ depending on $M^{\natural}, \lambda, \Lambda$, and $n$,

$$
\|u\|_{\mathrm{H}^{1}(\mathbb{R} ; H)}+\|u\|_{\mathrm{H}^{1 / 2}(\mathbb{R} ; V)} \leq C\|f\|_{\mathrm{L}^{2}(\mathbb{R} ; H)} .
$$

Proof. Let us first establish the required estimate under the additional qualitative assumptions that $f \in \mathrm{H}^{1}(\mathbb{R} ; H)$ and $A$ satisfies the Lipschitz condition. In this case Lemma $\left[5\right.$ guarantees the higher regularity $u \in \mathrm{H}^{1}(\mathbb{R} ; V)$. Hence $D_{t}^{1 / 2} u \in$ $\mathrm{H}^{1 / 2}(\mathbb{R} ; V) \subseteq \mathrm{E}$ and $D_{t}^{1 / 2} f \in \mathrm{H}^{1 / 2}(\mathbb{R} ; H) \subseteq \mathrm{E}^{*}$, which justifies the calculation

$$
D_{t}^{1 / 2} u=(1+\mathfrak{L})^{-1}\left(\mathfrak{L} D_{t}^{1 / 2}-D_{t}^{1 / 2} \mathfrak{L}\right) u+(1+\mathfrak{L})^{-1} D_{t}^{1 / 2} f
$$


as an equality in $\mathrm{E}$. For the first term we use $\mathfrak{L}=\partial_{t}+\nabla_{V}^{*} A \nabla_{V}$, so that having canceled the commutating terms,

$$
\left(\mathfrak{L} D_{t}^{1 / 2}-D_{t}^{1 / 2} \mathfrak{L}\right) u=\nabla_{V}^{*}\left(D_{t}^{1 / 2} A-A D_{t}^{1 / 2}\right) \nabla_{V} u .
$$

Since $\nabla_{V} u \in \mathrm{H}^{1}(\mathbb{R} ; H)^{n}$, we deduce from Corollary 7 the bound

$$
\begin{aligned}
\left\|\left(\mathfrak{L} D_{t}^{1 / 2}-D_{t}^{1 / 2} \mathfrak{L}\right) u\right\|_{\mathrm{L}^{2}\left(\mathbb{R} ; V^{*}\right)} & \leq\left\|\left(D_{t}^{1 / 2} A-A D_{t}^{1 / 2}\right) \nabla_{V} u\right\|_{\mathrm{L}^{2}(\mathbb{R} ; H)^{n}} \\
& \leq C M^{\natural}\left\|\nabla_{V} u\right\|_{\mathrm{L}^{2}(\mathbb{R} ; H)^{n}}
\end{aligned}
$$

Going back to (9), we now obtain from Lemma 4 that

$$
\begin{aligned}
\|u\|_{\mathrm{E}}+\left\|D_{t}^{1 / 2} u\right\|_{\mathrm{E}} & \leq \sqrt{2} \cdot \frac{\Lambda+1}{\lambda}\left(\|f\|_{\mathrm{E}^{*}}+\left\|\left(\mathfrak{L} D_{t}^{1 / 2}-D_{t}^{1 / 2} \mathfrak{L}\right) u\right\|_{\mathrm{E}^{*}}+\left\|D_{t}^{1 / 2} f\right\|_{\mathrm{E}^{*}}\right) \\
& \leq \sqrt{2} \cdot \frac{\Lambda+1}{\lambda}\left(2\|f\|_{\mathrm{L}^{2}(\mathbb{R} ; H)}+C M^{\natural}\|u\|_{\mathrm{L}^{2}(\mathbb{R} ; V)}\right) .
\end{aligned}
$$

Since $u=(1+\mathfrak{L})^{-1} f$, the same lemma yields the required estimate

$$
\leq 2 \sqrt{2} \cdot \frac{\Lambda+1}{\lambda}\|f\|_{\mathrm{L}^{2}(\mathbb{R} ; H)}+2 C M^{\natural}\left(\frac{\Lambda+1}{\lambda}\right)^{2}\|f\|_{\mathrm{L}^{2}(\mathbb{R} ; H)} .
$$

Now, we treat the general case by approximation. To this end let $\rho: \mathbb{R} \rightarrow[0, \infty)$ be smooth with compact support such that $\int_{\mathbb{R}} \rho(t) \mathrm{d} t=1$ and let $\rho_{n}(t)=n \rho(n t)$. Set $f_{n}:=\rho_{n} *_{t} f$ and $A_{n}:=\rho_{n} *_{t} A$, the symbol $*_{t}$ indicating the convolution on $\mathbb{R}$. Clearly $f_{n} \in \mathrm{H}^{1}(\mathbb{R} ; H)$ and $A_{n}$ satisfies the Lipschitz condition. Also

$$
A_{n}(t, x) \xi \cdot \bar{\zeta}=\int_{\mathbb{R}} \rho_{n}(t-s) A(s, x) \xi \cdot \bar{\zeta} \mathrm{d} s
$$

for all $\xi, \zeta \in \mathbb{C}^{n}$, where by construction $\rho_{n}$ is a positive functions with integral equal to 1 . Thus, these coefficients are bounded and coercive with the same parameters $\Lambda$ and $\lambda$ as is $A$. Similarly,

$$
\left\|D_{t}^{1 / 2} A_{n}(\cdot, x)\right\|_{\mathrm{BMO}(\mathbb{R})^{n \times n}}=\left\|\rho_{n} *_{t} D_{t}^{1 / 2} A(\cdot, x)\right\|_{\mathrm{BMO}(\mathbb{R})^{n \times n}} \leq M^{\natural}
$$

for a.e. $x \in \Omega$, using the translation invariance of the BMO-norm and the assumption on $A$ in the second step. So, if $u_{n}$ is the solution of

$$
u_{n}^{\prime}(t)+u_{n}(t)+\mathfrak{A}_{n}(t) u_{n}(t)=f_{n}(t) \quad(t \in \mathbb{R}),
$$

then from the first part of the proof we can infer that $\left\{u_{n}\right\}_{n}$ is a bounded sequence in $\mathrm{H}^{1}(\mathbb{R} ; H) \cap \mathrm{H}^{1 / 2}(\mathbb{R} ; V)$. Upon passing to a subsequence, we can assume that it has a weak limit $u \in \mathrm{H}^{1}(\mathbb{R} ; H) \cap \mathrm{H}^{1 / 2}(\mathbb{R} ; V)$. Given $v \in \mathrm{L}^{2}(\mathbb{R} ; V)$, we thus have

$$
\int_{\mathbb{R}}\left(u_{n}^{\prime}+u_{n}-f_{n} \mid v\right)_{H} \mathrm{~d} t=-\int_{\mathbb{R}}\left\langle\mathfrak{A}_{n} u_{n}, v\right\rangle \mathrm{d} t=-\int_{\mathbb{R}}\left(\nabla_{V} u_{n} \mid A_{n}^{*} \nabla_{V} v\right)_{H} \mathrm{~d} t
$$

and in order to reveal $u$ as the sought-after solution we have to pass to the limit $n \rightarrow \infty$.

This is easy for the left-hand side since $u_{n}^{\prime} \rightarrow u^{\prime}$ and $u_{n} \rightarrow u$ weakly and $f_{n} \rightarrow f$ strongly, all taking place in $\mathrm{L}^{2}(\mathbb{R} ; H)$. For the right-hand side we recall from Section 1.2 that $A$, when regarded as a function in $t$, is uniformly continuous with values in $\mathrm{L}^{\infty}\left(\Omega ; \mathbb{C}^{n \times n}\right)$. Hence, $A_{n}^{*}(t, x) \rightarrow A^{*}(t, x)$ for a.e. $(t, x) \in \mathbb{R} \times \Omega$. Eventually, the dominated convergence theorem tells us $A_{n}^{*} \nabla v \rightarrow \nabla v$ in $\mathrm{L}^{2}(\mathbb{R} ; H)$ and thus we can pass to the limit on the right-hand side, too. 
Remark 9. Recall that an equivalent formulation of the assumption in Proposition 8, more in the spirit of Theorem 2, has been discussed in Section 1.2.

Remark 10. A similar analysis can be performed for the homogeneous equation $u^{\prime}+\mathfrak{A} u=f$ on the real line, using a homogeneous version $\dot{\mathrm{E}}$ of the energy space, so that $\mathfrak{L}: \dot{\mathrm{E}} \rightarrow \dot{\mathrm{E}}^{*}$ becomes bounded and invertible. Under the assumption of Proposition 8 we formally obtain that for $f \in \mathrm{L}^{2}(\mathbb{R} ; H) \cap \dot{\mathrm{E}}^{*}$ the solution $u=\mathfrak{L}^{-1} f$ has higher regularity

$$
\left\|\partial_{t} u\right\|_{\mathrm{L}^{2}(\mathbb{R} ; H)}+\left\|\nabla_{V} D_{t}^{1 / 2} u\right\|_{\mathrm{L}^{2}(\mathbb{R} ; H)} \leq C\left(\|f\|_{\mathrm{L}^{2}(\mathbb{R} ; H)}+\|f\|_{\dot{\mathrm{E}}^{*}}\right) .
$$

The care of making this observation rigorous is left to the interested reader. It will not be needed in the following.

\section{The PRoOF OF THE MAIN RESUlT}

In this section we give the proof of Theorem 2 by reduction to the non-autonomous problem on the real line. This will mainly rely on the following extension lemma to the effect that an elliptic coefficient function satisfying the assumption of Theorem 2 can be extended to a function on $\mathbb{R} \times \Omega$ with half $t$-derivative in BMO.

Lemma 11. Suppose that $A:[0, T] \times \Omega \rightarrow \mathbb{C}^{n \times n}$ satisfies (17) and (8). Then $A$ can be extended to a map $A^{\natural}: \mathbb{R} \times \Omega \rightarrow \mathbb{C}^{n \times n}$ in such a way that the ellipticity bounds (17) remain true for all $t \in \mathbb{R}$ and

$$
\left\|D_{t}^{1 / 2} A^{\natural}(\cdot, x)\right\|_{\mathrm{BMO}(\mathbb{R})^{n \times n}} \leq M^{\natural} \quad(\text { a.e. } x \in \Omega)
$$

for some constant $M^{\natural}$ depending on $M, T, \Lambda, \lambda$, and $n$.

Proof. Due to Strichartz' characterization of BMO-Sobolev spaces [12, Thm. 3.3], it suffices to construct an extension $A^{\natural}: \mathbb{R} \times \Omega \rightarrow \mathbb{C}^{n \times n}$ with ellipticity bounds as in (7) and

$$
\int_{I} \int_{I} \frac{\left|A^{\natural}(t, x)-A^{\natural}(s, x)\right|^{2}}{|t-s|^{2}} \mathrm{~d} s \mathrm{~d} t \leq M^{\natural} \ell(I) \quad(\text { a.e. } x \in \Omega)
$$

for every interval $I \subseteq \mathbb{R}$, see also Section 1.2, Since the extension will be in $t$ direction only, we abbreviate $A(t, x)$ simply by $A(t)$. The proof is in two steps.

Step 1: Extension to $[-T, 2 T]$ by even reflection.

Let us define $A^{b}(t)=A(t)$ for $t \in[0, T]$ and $A^{b}(t)=A(-t)$ for $t \in[-T, 0]$. Clearly, this extension is elliptic with the same parameters as $A$. We claim that (11) holds for any interval $I \subseteq[-T, T]$. In fact, by assumption on $A$ we only have to treat the case $I=[-a, b]$, where $0 \leq a, b \leq T$. Here, we split

$$
\begin{aligned}
\int_{I} \int_{I} \frac{\left|A^{b}(t)-A^{\mathrm{b}}(s)\right|^{2}}{|t-s|^{2}} \mathrm{~d} s \mathrm{~d} t \leq & \left(\int_{0}^{a} \int_{0}^{a}+\int_{0}^{b} \int_{0}^{b}\right) \frac{|A(t, x)-A(s, x)|^{2}}{|t-s|^{2}} \mathrm{~d} s \mathrm{~d} t \\
& +2 \int_{0}^{a} \int_{0}^{b} \frac{|A(-t, x)-A(s, x)|^{2}}{|t+s|^{2}} \mathrm{~d} s \mathrm{~d} t
\end{aligned}
$$

where the first two integrals give a contribution of at most $M(a+b)=M \ell(I)$ and in the third integral we use $|t+s| \geq|t-s|$ to get a bound by $2 M \max \{a, b\} \leq 2 M \ell(I)$. Hence, $A^{b}$ satisfies the estimate with constant $3 M$. By the same procedure, we can further extend to $[-T, 2 T]$ by the expense of a constant $9 M$ in the estimate.

Step 2: Extension to the real line. 
We extend $A^{b}$ by zero outside of $[-T, 2 T]$. Then, we let $\varphi: \mathbb{R} \rightarrow[0,1]$ be equal to 1 on $[0, T]$, zero outside of $[-T / 2,3 T / 2]$, and connect continuously and linearly in between. The extension of $A$ that we consider is $A^{\natural}:=\varphi A^{b}+(1-\varphi) \lambda$.

Since $\varphi$ is independent of $x$, we easily see that $A^{\natural}$ satisfies the same ellipticity bounds as $A$. Concerning the estimate, we first bound the left-hand side of (11) by

$$
\int_{I} \int_{I} \varphi(t)^{2} \frac{\left|A^{b}(t)-A^{b}(s)\right|^{2}}{|t-s|^{2}} \mathrm{~d} s \mathrm{~d} t+\int_{I} \int_{I}\left(\left|A^{b}(s)\right|^{2}+\lambda^{2}\right) \frac{|\varphi(t)-\varphi(s)|^{2}}{|t-s|^{2}} \mathrm{~d} s \mathrm{~d} t .
$$

From the support properties of $\varphi$ and $A^{b}$ along with the bound $\left|A^{b}(t)\right| \leq \Lambda$ for a.e. $t \in \mathbb{R}$, we see that the first of these two integrals is no larger than

$$
\int_{I \cap\left[-\frac{T}{2}, \frac{3 T}{2}\right]} \int_{I \cap[-T, 2 T]} \frac{\left|A^{b}(t)-A^{b}(s)\right|^{2}}{|t-s|^{2}} \mathrm{~d} s \mathrm{~d} t+\int_{I \cap\left[-\frac{T}{2}, \frac{3 T}{2}\right]} \int_{I \backslash[-T, 2 T]} \frac{4 \Lambda^{2}}{|t-s|^{2}} \mathrm{~d} s \mathrm{~d} t .
$$

Thanks to the outcome of Step 1, this sums up to at most $\left(9 M+8 \Lambda^{2} T^{-1}\right) \ell(I)$. Similarly, for the second integral we obtain the upper bound $6\left(\Lambda^{2}+\lambda^{2}\right) T^{-1} \ell(I)$, using that $\varphi$ is bounded and Lipschitz continuous with constant $2 T^{-1}$.

Having all this at hand, the proof of Theorem 2 is rather routine.

Proof of Theorem . In the light of Lions' abstract result on maximal regularity in $V^{*}$ only the existence of a solution $u \in \mathrm{H}^{1}(0, T ; H) \cap \mathrm{H}^{1 / 2}(0, T ; V)$ is a concern. So, let $f \in \mathrm{L}^{2}(0, T ; H)$ be given and let $E_{0} f \in \mathrm{L}^{2}(\mathbb{R} ; H)$ be its extension by zero. Also, we extend $A$ to the whole real line using Lemma [1], where for convenience this extension is also denote by $A$. According to Proposition 8 there exists a solution $u \in \mathrm{H}^{1}(\mathbb{R} ; H) \cap \mathrm{H}^{1 / 2}(\mathbb{R} ; V)$ to the problem

$$
u^{\prime}(t)+u(t)+\mathfrak{A}(t) u(t)=\mathrm{e}^{t} E_{0} f(t) \quad(t \in \mathbb{R}) .
$$

Once we have checked $u(0)=0$, the restriction of $\mathrm{e}^{-t} u(t)$ to $[0, T]$ will be the solution we are looking for and the precise estimate stated in the theorem is a consequence of Proposition 8 and Lemma 11.

Since $u \in \mathrm{H}^{1}\left(\mathbb{R} ; V^{*}\right) \cap \mathrm{L}^{2}(\mathbb{R} ; V)$, the function $\|u\|_{H}^{2}$ is absolutely continuous with derivative $\frac{\mathrm{d}}{\mathrm{d} t}\|u\|_{H}^{2}=2 \operatorname{Re}\left\langle u^{\prime}, u\right\rangle$. (Check this for smoothened versions of $u$ first). Thus,

$\min \{1, \lambda\} \int_{-\infty}^{0}\|u\|_{V}^{2} \mathrm{~d} t \leq \operatorname{Re} \int_{-\infty}^{0}\langle u+\mathfrak{A} u, u\rangle \mathrm{d} t=-\operatorname{Re} \int_{-\infty}^{0}\left\langle u^{\prime}, u\right\rangle \mathrm{d} t=-\frac{1}{2}\|u(0)\|_{H}^{2}$,

where we have used the equation for $u$ along with $E_{0} f=0$ on $(-\infty, 0)$ in the second step. Thus, $\|u(0)\|_{H}=0$.

Remark 12. A combination of Lemma 4 and the argument performed above gives a new, neat proof of Lions' abstract maximal regularity result in $V^{*}$ itself, assuming only boundedness, quasi-coercivity, and measurability of $\mathfrak{a}$. In particular, $H$ does not have to be separable. This was done by Dier and Zacher, see [3, Thm. 6.1].

\section{FURTHER REMARKS AND OPEN QUESTIONS}

Let us revisit the proof of Theorem 2 . Given $f \in \mathrm{L}^{2}(\mathbb{R} ; H)$, Lemma 4 gave us a a solution $u$ to the non-autonomous problem $u^{\prime}+u+\mathfrak{A} u=f$ on the real line that additionally satisfied $D_{t}^{1 / 2} u \in \mathrm{L}^{2}(\mathbb{R} ; H)$. Then, it was the boundedness of the commutator $\left[A, D_{t}^{1 / 2}\right]$ on $\mathrm{L}^{2}(\mathbb{R} ; H)$ that followed from our assumption on $\mathfrak{A}$ and in turn presented us with one-half derivative more in $\mathrm{L}^{2}(\mathbb{R} ; H)$, that is, maximal 
regularity. With this at hand, the proof could be completed by rather standard arguments.

Analogous commutator bounds hold for any fractional derivative $D_{t}^{\alpha}, \alpha \in(0,1)$, see again [7, Thm. 3.3] or use the $T(1)$-theorem. The following result can therefore be obtained by a literal repetition of the argument. Results of the same spirit also appeared in [3, Thm. 6.2] for general non-autonomous forms under different regularity assumptions.

Theorem 13. Consider a non-autonomous form $\mathfrak{a}$ inducing elliptic divergenceform operators with either Dirichlet, Neumann, or mixed boundary conditions on an open set $\Omega \subseteq \mathbb{R}^{n}$ as defined in Section 1.1. Suppose there exist $\alpha \in\left(0, \frac{1}{2}\right)$ and $M \geq 0$ such that

$$
\left.\sup _{I \subseteq[0, T]} \frac{1}{\ell(I)} \int_{I} \int_{I} \frac{|A(t, x)-A(s, x)|^{2}}{|t-s|^{1+2 \alpha}} \mathrm{d} s \mathrm{~d} t \leq M \quad \text { (a.e. } x \in \Omega\right) .
$$

Then, given $f \in \mathrm{L}^{2}(0, T ; \mathrm{H})$, the unique solution $u \in \mathrm{H}^{1}\left(0, T ; V^{*}\right) \cap \mathrm{L}^{2}(0, T ; V)$ of problem (2) satisfies

$$
\|u\|_{\mathrm{H}^{\alpha+1 / 2}(0, T ; H)}+\|u\|_{\mathrm{H}^{\alpha}(0, T ; V)} \leq C\|f\|_{\mathrm{L}^{2}(0, T ; H)},
$$

where $C$ depends on $\lambda, \Lambda, \alpha, M, T$, and $n$.

Finally let us recall that Murray's commutator estimate from Proposition 6 is sharp. In particular, it does not remain true if the multiplier is merely $\frac{1}{2}$-Hölder continuous [7. Also the proof of Proposition 8 supplies an exact factorization of the half time-derivative of the solution using the commutator $\left[A, D_{t}^{1 / 2}\right]$, compare with equations (9) and (10). Guided by this, we make the following

Conjecture 14. The $\frac{1}{2}$-Hölder continuity of $\mathfrak{A}:[0, T] \rightarrow \mathcal{L}\left(V, V^{*}\right)$, that is,

$$
\|\mathfrak{A}(t)-\mathfrak{A}(s)\|_{V \rightarrow V^{*}} \leq C|t-s|^{1 / 2} \quad(t, s \in[0, T]),
$$

does not imply maximal regularity of $\mathfrak{a}$ in $H$ in general, not even if $\mathfrak{a}$ induces elliptic differential operators in divergence form with Dirichlet, Neumann, or mixed boundary conditions on some open set $\Omega$.

\section{REFERENCES}

[1] R. Dautray and J.L. Lions. Mathematical Analysis and Numerical Methods for Science and Technology - Volume 5 Evolution Problems I. Springer-Verlag, Berlin, 1992.

[2] G. David and J.L. Journé. A boundedness criterion for generalized Calderón-Zygmund operators. Ann. of Math. (2) 120 (1984), no. 2, 371-397.

[3] D. Dier and R. ZACher. Non-autonomous maximal regularity in Hilbert spaces. Available at http://arxiv.org/abs/1601.05213

[4] S. FACKLER. J.L. Lions' problem concerning maximal regularity of equations governed by non-autonomous forms. Available at http://arxiv.org/abs/1601.08012

[5] B.H. HAAK and E.M. OUHABAz. Maximal regularity for non-autonomous evolution equations. Math. Ann. 363 (2015), no. 3, 1117-1145.

[6] J.L. Lions. Équations différentielles opérationnelles et problèmes aux limites. Die Grundlehren der mathematischen Wissenschaften, vol. 111, Springer-Verlag, BerlinGöttingen-Heidelberg, 1961.

[7] M.A.M. Murray. Commutators with fractional differentiation and BMO Sobolev spaces. Indiana Univ. Math. J. 34 (1985), no. 1, 205-215.

[8] K. Nyström. Square functions estimates and the Kato problem for second order parabolic operators in $\mathbb{R}^{n+1}$. Advances in Mathematics 293 (2016), 1-36. 
[9] E.M. Ouhabaz and C. SpinA. Maximal regularity for non-autonomous Schrödinger type equations. J. Differential Equations 248 (2010), no. 7, 1668-1683.

[10] L. DE Simon. Un'applicazione della teoria degli integrali singolari allo studio delle equazioni differenziali lineari astratte del primo ordine. Rend. Sem. Mat. Univ. Padova 34 (1964), 205-223.

[11] E.M. Stein. Singular Integrals and Differentiability Properties of Functions. Princeton Univ. Press, Princeton, 1970.

[12] R.S. Strichartz. Bounded mean oscillation and Sobolev spaces. Indiana Univ. Math. J. 29 (1980), no. 4, 539-558.

[13] H. Vogt. Equivalence of Pointwise and Global Ellipticity Estimates. Math. Nachr. 237 (2002), no. 1, 125-128.

Laboratoire de Mathématiques d'Orsay, Univ. Paris-Sud, CNRS, Université ParisSaclay, 91405 Orsay, France

E-mail address: pascal.auscher@math.u-psud.fr, moritz.egert@math.u-psud.fr 